\title{
Conversion to Parkinsonism and Dementia in REM-Sleep Behavior Disorder Using the Chronobiotic Melatonin
}

\author{
Dieter Kunz, $\mathrm{MD}^{1,2}$, Sophia Stotz, $\mathrm{MSc}^{1,2}$ and Frederik Bes, $\mathrm{PhD}^{1,2}$ \\ ${ }^{1}$ Clinic for Sleep- \& Chronomedicine, St. Hedwig-Krankenhaus Berlin, Germany \\ ${ }^{2}$ Charité Universitätsmedizin Berlin, Institute of Physiology, Berlin, Germany
}

\begin{abstract}
Background: Isolated REM sleep behavior disorder (iRBD), a reliable prodromal stage marker of $\alpha$-synucleinopathies like Parkinson's disease or Lewy body dementia, offers an early window for disease-modifying intervention. Current treatments of $\mathrm{iRBD}$, including the two level B therapies with clonazepam and melatonin, are considered symptomatic. However, numbers of reported patients treated with melatonin are low and whether melatonin has disease-modifying potential is unclear.
\end{abstract}

Methods: This single-center, prospective cohort study included 206 consecutive patients diagnosed with iRBD until January 2020. Thirty-nine patients had applied mixed treatments on the advice of the referring physician, 167 had administered melatonin according to our chronobiotic protocol (low dose, $\geq 6$ months, always-at-the-same-clock-time, between 10 and $11 \mathrm{pm}$ - corrected for chronotype), which differs from existing melatonin prescriptions. Clinical examination to determine phenoconversion was performed from October 2018 to August 2020. To evaluate generalizability, we compared factors such as neuropsychological and neuromotor performance, olfactory ability, neurovegetative behavior, and dopamine transporter density in our patients with those reported for other cohorts. Primary outcome was phenoconversion to clinical synucleinopathy, assessed using Kaplan-Meier analysis. Secondary outcomes were changes in cognitive and motor performance, and in RBDsymptom severity, analyzed using mixed models.

Results: RBD characteristics were comparable to those in other published cohorts, including frequency of phenoconversion in our patients with mixed treatments (10/39; follow-up $3.1 \pm 2.1$ years). In contrast, long-term melatonin-treated patients rarely converted (4/167; follow-up $4.2 \pm 3.1$ years; hazard-ratio $0.07,95 \% \mathrm{CI}, 0.02-0.22, \mathrm{p}<0.001)$. Neuromotor and 
medRxiv preprint doi: https://doi.org/10.1101/2020.11.05.20224592; this version posted November 12, 2020. The copyright holder for this

neuropsychological performance did not decline, improved in some domains. Symptom severity gradually improved over the first 4 weeks of treatment (Clinical Global Impression Severity: 5.7 vs. 3.0 ) and remained stable over years, also in those patients who had stopped melatonin intake after 6 months. The initial response was slower in patients with melatonin suppressing (beta blockers) or REM sleep spoiling co-medication (antidepressants) and failed with inadequate timing of melatonin intake.

Conclusion: Clock-timed melatonin treatment in patients with iRBD appears to be associated with a marked reduction in the development of parkinsonism and dementia as well as with an improvement in neuromotor, cognitive, and specific RBD symptoms. Findings suggest that melatonin treatment may have disease-modifying effects in synucleinopathies. The fact that melatonin is available anywhere at low cost provides the perspective of immediate clinical application in patients at risk for clinical synucleinopathy. On the other hand, clock-time dependency challenges existing prescription guidelines for melatonin. Melatonin should be acknowledged as the darkness signal to circadian clock-work rather than a hypnotic.

\section{INTRODUCTION}

Parkinsonism and dementia caused by neurodegeneration are characterized by gradual, irreversible worsening of physical and mental abilities, leading to patient distress and dysautonomy, severe burden for care givers, and high socio-economic costs (GBD, 2016). Knowledge of the disease has improved substantially in recent years, and effective symptomatic treatment has been identified, improving patients' quality of life. Attempts to modify the disease, however, have not been successful to date (Espay et al., 2017, 2020). The clinical signs of parkinsonism and dementia occur when the underlying neurodegenerative process is already far advanced (Braak et al., 2003). Hence, a major challenge of research in this field is to identify prodromal biomarkers to aid early diagnosis, to monitor disease progression, and to find a means for preventive treatment (Postuma and Berg, 2019; Videnovic et al., 2020).

Isolated rapid-eye-movement sleep behavior disorder (iRBD) is recognized as the most reliable prodromal biomarker of synucleinopathies such as Lewy body dementia (LBD) and Parkinson's disease (Schenck et al., 2013; Iranzo et al., 2014; Postuma et al., 2019). In patients with $\mathrm{RBD}$, the characteristic atonia of voluntary muscles in rapid-eye-movement (REM) sleep is impaired, leading to the acting out of dreams. Initially, patients start to 
medRxiv preprint doi: https://doi.org/10.1101/2020.11.05.20224592; this version posted November 12, 2020. The copyright holder for this

vocalize, speak, and move complexly. As the disease progresses, patients may yell, fight, or jump out of bed, often injuring themselves or their sleeping partner (Schenck et al., 1986).

The mainstay of treatment in RBD is the prevention of injuries by modifying patients' sleep environment and suppressing motor activity during sleep with muscle relaxants, usually clonazepam (Iranzo et al., 2016; Videnovic et al., 2020). Clonazepam immediately improves motor behavior during the first night of treatment. Being reluctant to initiate a long-term benzodiazepine treatment we introduced the use of melatonin as a therapy for patients with RBD (Kunz and Bes, 1997, 1999). Four common observations found in three different patient populations (Kunz and Bes, 1999, 2001; Kunz et al., 2004; Kunz and Mahlberg, 2010) pointed towards the circadian clock to be involved in the mode of action: 1. response occurred only when melatonin was administered within a narrow time span between 10 and $11 \mathrm{pm} ; 2$. responders and non-responders were best differentiated by a stable clock-time of administration as opposed to flexible administration (e.g. "at bedtime" or "after meal"); 3 . gradual response over weeks; 4. persisting effect after discontinuation of treatment. Based on these observations, we have developed a "chronobiotic protocol" for the use of melatonin treatment (Kunz, 2004). All our patients to be treated with melatonin are advised to follow this protocol since then. Although these effects may seem peculiar, melatonin is nowadays considered a first-line treatment in RBD, like clonazepam (Aurora et al., 2010; Videnovic et al., 2020).

Whereas reported rates of phenoconversion to clinical synucleinopathy rely on cohorts with a mixture of treatment strategies, primarily clonazepam (Fernandez-Arcos et al., 2016; Postuma et al., 2019; Galbiati et al., 2019), no large dataset has been reported for patients with iRBD treated with melatonin only. In this prospective cohort study, we aimed to determine the frequency of phenoconversion in two cohorts of patients with iRBD, one treated with the usual mixture of treatments and one with melatonin according to the chronobiotic protocol.

\section{Materials and methods}

\section{Patients}

Until August 2020 we diagnosed RBD in 282 consecutive patients according to the International Classification of Sleep Disorders (ICSD-3) using three night's videopolysomnography (vPSG). Isolated RBD was present in 218 patients of whom 12 were diagnosed recently and by consequence, a follow-up examination was not yet performed (Fig. 
medRxiv preprint doi: https://doi.org/10.1101/2020.11.05.20224592; this version posted November 12, 2020. The copyright holder for this

1). Of the 206 patients evaluated, 167 patients were treated with melatonin according to our chronobiotic protocol, 39 were treated with the usual mixture of treatments.

In our clinic, most patients are referred by a neurologist / general practitioner or contact us personally. They are diagnosed and redirected with a therapeutic recommendation. In patients with RBD we usually initiate treatment and patients are advised to administer melatonin on a long-term basis. In most of RBD patients our patient care stops, when symptoms are resolved. In those patients, who are reluctant towards medication, we used to recommend administration of melatonin for at least 6 months. Even though long-term melatonin treatment had been recommended to all of our patients, $39 \mathrm{iRBD}$ patients had not been treated with melatonin for 6 or more months ("mixed treatment" group). This was because they discontinued melatonin therapy after concluding that "they were cured" because RBD symptoms did not reoccur after discontinuation of melatonin up to 3 months ( $\mathrm{n}=13$, "shortterm melatonin" in Fig. 4). The remaining 26 patients ("various" in Fig. 4) experienced an initial worsening or treatment failure (melatonin stopped after 1-7 nights; $n=16$ ), and were hesitant to take melatonin or their treating physicians were reluctant to prescribe it (melatonin was stopped within 4 weeks; $n=10$ ). In those 26 patients the usual mixture of therapies was applied by their neurologist / general practitioner (e.g. clonazepam, other benzodiazepines, zolpidem, dopaminergic agents, mirtazapine, trazodone or no treatment).

The RBD-specific characteristics of our patients were compared in tabular form (Table 1) with three different kinds of representative samples (Fernandez-Arcos et al., 2016; Postuma et al., 2019; Galbiati et al., 2019).

\section{Chronobiotic Protocol}

In our clinic we offer patients clinically suspected to have RBD to pass through a three night's vPSG. Prior to the third night, we advise them to initiate a long-term melatonin treatment according to a chronobiotic protocol, which implies one major instruction: to administer melatonin "always-at-the-same-clock-time" once per day. This clock time should be established at 30 minutes prior to patient's habitual bedtime. In cases where this fixed time cannot be kept, patients are instructed to skip melatonin intake that night. The rationale for this strict schedule is that since our initial pilot studies with RBD patients, we repeatedly observed that responders and non-responders were best distinguished by evaluating their sleep hygiene, i.e. stable vs. varying bedtimes and times of melatonin intake (summarized in Kunz, 2004). This clinical observation is in agreement with the fact that melatonin is known to feed 
medRxiv preprint doi: https://doi.org/10.1101/2020.11.05.20224592; this version posted November 12, 2020. The copyright holder for this

back on the suprachiasmatic nucleus, the central pacemaker or masterclock (Gilette and McArthur, 1996; Gerdin et al., 2004). As a consequence, exogenous melatonin should be administered consistently within a rather narrow time span in order to gain optimal effects. Patients are informed that melatonin in RBD rarely exhibits effects during first days of treatment, rather effects occur within the first two weeks. Sometimes symptoms even worsen over the first days of administration, presumably because of transient initial sleep disruption due to immediate chronobiotic effects of melatonin, i.e. delaying or advancing the patient's actual circadian phase (Lewy et al., 1992). In those patients, in whom melatonin does not show positive effects over the first three weeks of treatment, the time of administration is controlled referring to individual chronotype, e.g. in case of a mismatch with habitual bedtime.

\section{RBD Test Battery}

When findings began to spread on the internet that iRBD represents a prodromal stage of clinical synucleinopathy, patients with RBD symptoms sought information on which stage of prodromal synucleinopathy they might be in. Since the end of 2015, we have therefore offered to all our new RBD patients a battery of examinations consisting of neurocognitive testing (CERAD-Plus, Schmid et al., 2014), neurologic/motoric evaluation of Parkinson's severity (MDS-UPDRS-part III, Goetz et al., 2008), neurovegetative questioning (SCOPA-AUT, Visser et al., 2004), olfaction testing (Sniffin' Sticks, Hummel et al., 1997), and dopamine transporter single-photon-emission computed tomography (DaT-SPECT, Djang et al., 2012)). CERAD-Plus, UPDRS-III, and SCOPA-AUT may be repeated annually. Over time, we expanded this initiative also to former patients being already under melatonin treatment $(\mathrm{n}=50)$, when they contacted us for follow-up.

Starting October 2018, we contacted all of our patients with iRBD for the evaluation of phenoconversion to clinical synucleinopathy. For treatment response, we specifically developed an RBD-symptoms severity rating scale called Ikelos-RS, after the god and personification of nightmares in the Greek mythology (inter-rater reliability: (correlation, Spearman's rho) $\rho=0.9, \mathrm{p}<0.001$; test-retest reliability: $\rho=0.9, \mathrm{p}<0.001$; further details see Supplementary Methods).

All patients had provided written informed consent for their clinical data to be analyzed and published anonymously. The ethics committee of Charité - Universitätmedizin Berlin approved publication of the results of the post-hoc data analysis. 
medRxiv preprint doi: https://doi.org/10.1101/2020.11.05.20224592; this version posted November 12, 2020. The copyright holder for this

\section{Outcomes}

The primary outcome was phenoconversion to clinical synucleinopathy after PSG-confirmed diagnosis, in a time-to-event analysis. Data for 206 evaluated patients were censored at last follow-up 2018 to $2020(\mathrm{n}=183)$, or at the time when a patient was diagnosed as having phenoconverted $(\mathrm{n}=14)$, had died $(\mathrm{n}=8)$, or was lost to follow-up $(\mathrm{n}=1)$. Phenoconversion was diagnosed at our site by means of a clinical neurological evaluation (criteria of Movement Disorders Society), supported by CERAD-Plus and UPDRS-III (performed in 141, respectively 153 of 167 patients under long-term melatonin treatment). Additionally, whenever the referring neurologists initiated specific treatment for parkinsonism/dementia, this was considered to be due to phenoconversion. Eight of 167 treated patients had died, and 11 could not be reached in person. In these 19 cases, spouses and referring physicians were contacted and asked if the patients had shown signs of parkinsonism/dementia. In only one patient, follow-up during prospective evaluation of phenoconversion after October 2018 was not possible. The last available follow-up was in 2017, five years after diagnosis, because the family had moved to an unknown new place of residence.

Secondary outcomes were variations over time in initial and follow-up scores on cognition (CERAD-Plus), neuromotor performance (UPDRS-III) and RBD symptom severity (IkelosRS, Clinical Global Impression (CGI)). In total, 1489 Ikelos-RS and CGI, 396 CERAD-Plus and 406 UPDRS-III scores were examined. Statistical analyses included only data from patients who at baseline had not yet taken melatonin and who underwent at least one followup examination during further treatment.

To analyze changes in RBD symptom severity over the first 6 months of treatment in more detail (Fig. 4), patients treated according to the chronobiotic protocol were classified into four subgroups according to known confounding factors with melatonin intake: use of beta blockers, use of antidepressants, melatonin intake not always-at-the-same-clock-time, and absence of these confounding factors. For analysis after this initial period, patients were reclassified into 2 subgroups, one with continued melatonin intake, and one with patients who stopped or interrupted melatonin at or shortly after this time. Those 39 patients being treated with the usual mixture of therapies were classified in two groups: one with positive initial response ("short-term melatonin"; improvement $\geq 2$ on Ikelos-RS and CGI-S; $n=13$ ) during melatonin treatment over 1 to 3 months (feeling "cured") and one without positive response 
medRxiv preprint doi: https://doi.org/10.1101/2020.11.05.20224592; this version posted November 12, 2020. The copyright holder for this

("various"; $\mathrm{n}=26)$ during less than 4 weeks melatonin treatment $(\mathrm{n}=16)$ or no melatonin treatment $(\mathrm{n}=10)$.

\section{Statistical analysis}

Phenoconversion to clinical synucleinopathy was investigated in groups with or without longterm melatonin treatment using Kaplan-Meier survival analysis and the log-rank test. Hazard ratios and 95\% confidence intervals were calculated using a Cox proportional-hazards model. In patients treated with melatonin, variation in cognitive (CERAD-Plus) and motor (UPDRSIII) performance over time was tested using linear mixed models. Model 1 analyzed each subtest (using z-scores) as dependent variable, treating "time category" as a fixed factor, "patient ID" as a random factor, and "continuous vs. discontinuous melatonin intake" and "normal vs. borderline/pathological DaTSPECT" as covariates. Based on the hypothesis that the clinically most impaired patients benefit most from treatment, subtests with significant results were categorized into low, medium, and high performers at baseline. This group factor was analyzed in model 2. Variations over time in RBD symptom severity (Ikelos-RS), both within and between subgroups, were analyzed using a random intercept model. Bonferroni post-hoc test was used for multiple testing corrections in all executed mixed models. Significance was defined as p-values less than 0.05. Analyses and modeling were implemented using IBM SPSS Statistics (version 23.0). Details of these analyses are provided in the supplementary material.

\section{RESULTS}

A comparison of the RBD specifics of our patients with those of three representative cohorts suggests that our results are generalizable (Table 1). A remarkable exception, however, is the rate of phenoconversion to clinical synucleinopathy in our patients on long-term melatonin treatment according to chronobiotic protocol, which was extremely low: only 4 out of 167 patients $(2.4 \%)$ converted after a mean follow-up of $4.2 \pm 3.1$ years, implying a conversion rate of $0.6 \% / y e a r$. This is in sharp contrast to the phenoconversion rate in a) our 39 patients on mixed therapies, of whom $10(25.6 \%)$ converted after a mean follow-up of $3.1 \pm 2.1$ years, yielding a conversion rate of $8.3 \%$ year and $b$ ) the three representative patient cohorts with mixed therapies used for comparison, in which the conversation rate ranged from $6 \%$ to 7\%/year (Tab. 1, Fig. 2). In our cohort, treatment with melatonin for at least 6 months was associated with a reduced hazard of conversion of 0.07 (95\% CI, 0.02-0.22; $<<0.001$; Supplementary Tab. 1). 
medRxiv preprint doi: https://doi.org/10.1101/2020.11.05.20224592; this version posted November 12, 2020. The copyright holder for this

Moreover, cognitive performance - available from 91 patients before long-term melatonin treatment - did not decline over time (Fig. 3; Supplementary Tab. 2) and even improved on several subtests, with significant main effects seen for "Wordlist Encoding - verbal memory" $\left(\mathrm{F}_{3,133}=7.33, \mathrm{p}<0.001\right.$; Supplementary Tab. 3) and "Trail Making Test - Part A (TMT-A) visual attention performance" $\left(\mathrm{F}_{3,130}=5.02, \mathrm{p}=0.003\right.$; Supplementary Tab. 4). In subtests for which significant results were observed, categorizing our cohort into low, medium, and high performers at baseline showed that low and medium performers improved over time in verbal memory $\left(\mathrm{F}_{6,140}=4.94, \mathrm{p}<0.001\right.$; Supplementary Tab. 5) and visual attention $\left(\mathrm{F}_{6,135}=4.19\right.$, $\mathrm{p}=0.001$; Supplementary Tab. 6), with low performers tending to achieve cognitive levels that were clinically inconspicuous (Fig. 3). Of the 16 patients with a mild neurocognitive disorder (NCD) at baseline, none had developed dementia or parkinsonism by the time of their last follow-up, two had worsened, three remained stable, and 11 had improved cognition. Of these 11 patients, eight did not fulfill the criteria for an NCD at last follow-up anymore. Of the remaining 151 patients without NCD at baseline, none developed an NCD during or after melatonin treatment.

Neuromotor performance measured with UPDRS-III in 75 patients without melatonin at baseline (Supplementary Tab. 7) did not decline over time on long-term melatonin treatment either, but improved: the mean baseline score of 2.0 improved to 1.1 after 0.9 years and to 0.9 after 1.7 years, remaining stable at 0.7 after 3.5 years $\left(F_{3,104}=9.81, p<0.001\right.$; Supplementary Tab. 8). Again, the clinically most impaired performers improved more pronounced, with a mean total score of 6.6 at baseline and of 2.3 after 3.5 years $\left(\mathrm{F}_{6,103}=13.33\right.$, p $<0.001$; Supplementary Tab. 9). The covariates "continuity of melatonin intake" and "DaTSPECT classification" did not explain results in CERAD-Plus or UPDRS-III.

Fig. 4 shows that patients, who administered melatonin according to our chronobiotic protocol improved over first 4 to 6 weeks of treatment and remained stably improved. In contrast, patients administering melatonin, but not always at the same clock time, initially failed to show improvements in RBD symptom severity $\left(\mathrm{F}_{5,1420}=10.25, \mathrm{p}<0.001\right.$; Supplementary Tab. 10). Eleven of these patients reported an initial worsening of RBD symptoms during the first few days up to one week after starting melatonin treatment due to misinterpretation of the ambiguous prescription in the medication package leaflet to "administer melatonin after a meal, 1-2 hours before bedtime". This resulted in intake that was too early, around 6 to $8 \mathrm{pm}$ (i.e. after dinner). The remaining 14 patients in this category varied their time of intake by several hours, linking it to changing bedtimes. After instructions on proper timing were given 
medRxiv preprint doi: https://doi.org/10.1101/2020.11.05.20224592; this version posted November 12, 2020. The copyright holder for this

again, RBD symptoms improved in all of these patients. Patients on concomitant beta blocker or antidepressant therapy responded more slowly and with less pronounced effects in the beginning. RBD symptoms leveled out at low Ikelos-RS values, with almost no patient reporting a complete disappearance of RBD symptoms. Improvement in Ikelos-RS was paralleled by a change in CGI severity $(\rho=0.8, p<0.001)$. Of 167 patients on long-term melatonin treatment, 137 took melatonin at least $50 \%$ of the time between treatment initiation and last follow-up (max. 21.7 years), and 30 discontinued after 6 months. RBD symptoms did not worsen over time $\left(\mathrm{F}_{1,812}=1.30, \mathrm{p}=0.254\right.$; Supplementary Tab. 11) in any of these patients, including those who discontinued treatment after 6 months (Fig. 4, right panel).

In the cohort of patients on mixed treatments acting out of dreams improved in 13 patients (Fig. 4; "short-term melatonin") and administration of melatonin was stopped after 1 to 3 months because patients felt "cured" and considered a long-term medication with melatonin to be unnecessary. Symptoms partly returned in frequency and severity in follow-up and five patients converted $\left(\mathrm{F}_{3,782}=3.57, \mathrm{p}=0.014\right.$; Supplementary Tab. 12). Those patients without an initial improvement (Fig. 4, "various") did not report changes in frequency or severity of RBD symptoms over time (e.g. a wearing-off of RBD symptoms).

\section{DISCUSSION}

The main finding of our study is an exceptionally low rate of conversion to parkinsonism and dementia in the group of patients with iRBD who were under long-term melatonin treatment according to our chronobiotic protocol. With the seminal publication by Schenck et al. in 1996, initial evidence became available that iRBD was somehow linked to parkinsonism. Today it is recognized that $\mathrm{RBDD}$ represents the most reliable marker of prodromal state in the neurodegenerative process leading to clinical synucleinopathies, with a stable phenoconversion rate of $6 \%$ to 7\% per year (Fernandez-Arcos et al., 2016; Postuma et al., 2019; Galbiati et al., 2019). Thus, the number of converters among 167 patients with iRBD over 4.2 years would be expected to have been 40 to 50 , but in our cohort of patients we diagnosed only four, with either Parkinson`s disease or Lewy body dementia.

This result for our primary outcome is well supported by the results for our secondary outcome variables. Cognitive performance and motor functioning decline with healthy aging, and this process is accelerated with neurodegeneration (Terzaghi et al., 2013; Darweesh et al., 
medRxiv preprint doi: https://doi.org/10.1101/2020.11.05.20224592; this version posted November 12, 2020. The copyright holder for this

2017). Our patients treated with melatonin, however, not only did not experience a decline; they even improved in neuromotor and some subdomains of cognitive performance. Improvements were most pronounced in low performers (including patients diagnosed with NCD at baseline), who tended to reach performance levels that were near normal. Most interestingly, attention, which has been identified as a first-line cognitive indicator of developing LBD (Genier-Marchand et al., 2017), was significantly improved in low and medium performers. Assuming that the underlying neurodegenerative process has started at least at the onset of RBD and is already well underway, it is worth noting that in melatonin treated patients performance in nearly all cognitive subtests of the CERAD-Plus was on average still clearly above representative norm levels (i.e., z-score of zero) after many - here $8.7 \pm 5.3$ - years after reported onset of RBD-symptoms.

All of our patients presented at baseline with involuntary falling out of bed or having attacked their bed partner (indicating a high severity of RBD symptoms) in the 3 months before RBD diagnosis. Within 2 weeks after starting melatonin treatment, however, while bed partners still noticed complex behavior, aggressive behavior had almost disappeared, which was objectivated by substantial improvement in Clinical Global Impression and RBD symptom severity. Improvements in RBD symptoms persisted for years even when melatonin was discontinued. This long-lasting improvement cannot be attributed to a wearing off of symptoms over time. Our group of patients being treated with the usual mixture showed no improvement over time. The time of treatment necessary for melatonin remains unclear and can only be estimated. Whereas patients who stopped melatonin administration after at least 6 months remained improved, patients who discontinued melatonin after 4 to 12 weeks worsened again with respect to RBD symptoms - though not to baseline levels - and converted at a similar rate to clinical synucleinopathy as those not treated according to a chronobiotic protocol.

Three aspects need to be considered when interpreting our findings: their generalizability, the mode of action of melatonin, and the limitations of our study.

Generalizability. Our cohort represents the largest single center cohort on iRBD reported thus far and is comparable to other published cohorts with respect, first, to all major characteristics of patients with RBD (e.g. sex, age of onset of RBD symptoms, RBD severity, co-morbidities, co-medications) and, second, to olfactory deficit, abnormal motor symptoms, cognitive impairment, and abnormal DaT-SPECT, which have been identified as markers of 
medRxiv preprint doi: https://doi.org/10.1101/2020.11.05.20224592; this version posted November 12, 2020. The copyright holder for this

an advanced state in the phenoconversion of patients with iRBD (Iranzo et al., 2017; Postuma et al., 2019). Third, in patients in our cohort who had not been treated with melatonin according to our chronobiotic protocol over the long term, the conversion rate was as one would expect from published data of patients with iRBD.

Mode of Action. The present co-first line treatment for RBD, clonazepam, immediately improves RBD-related motor behavior, but the symptoms return within two nights after discontinuation (Fernandez-Arcos et al., 2016). In contrast, melatonin is not known to have any direct effects on motor behavior. Moreover, symptoms remain improved long after discontinuation of treatment, sometimes after years in those patients who stop administration of melatonin. Neuroprotective effects via melatonin (Reiter et al., 2013; Cardinali, 2019) and its receptors (Jockers et al., 2016) are to be studied.

The present results and our experience with melatonin indicate the known chronobiotic effects of melatonin which are highly time-dependent. Exogenous melatonin shifts the internal circadian phase according to a phase response curve and can be used in jetlag or to adjust late chronotypes to social time schedules (Lewy et al., 1992). Crucial in the context of RBD, however, might be a second, yet under-appreciated chronobiotic effect of melatonin: its darkness promoting property (Utiger, 1992; Dawson and Armstrong, 1996). When administered during its endogenous rise in the evening, melatonin does not shift phase, but strengthens the coordination of nightly circadian factors, with consequences such as promoting wake-related activities in nocturnal species and sleep-related activities in diurnal species. The sleep stage predominantly driven and modulated by the circadian timing system is that of REM sleep (Dijk et al., 1995; Bes et al.; 1996; Saper et al., 2005). The timing of impulses to this system seems decisive and, once established, needs to be kept "always-at-thesame-clock-time". In contrast, administering melatonin at an inappropriate time induces phase shifts resulting in desynchrony of internal rhythms (Samel et al., 1991; Monk et al., 2000). Some of our patients reported initial worsening of RBD symptoms (e.g. jumping out of bed 3 nights in a row), most likely due to inadequate timing with respect to their individual circadian phase. Of the four patients who phenoconverted despite long-term melatonin treatment, one administered melatonin strictly at bedtime, which included around 10 days per month melatonin at 8 a.m. because of daytime sleep after nightshift work. Another patient who converted shifted time of administration once per week for 3 hours due to occupational constraints. Inadequate timing of melatonin seems likely not only to fail in improvement, but rather seems to worsen symptomatology due to desynchronization. Those patients who 
medRxiv preprint doi: https://doi.org/10.1101/2020.11.05.20224592; this version posted November 12, 2020. The copyright holder for this

properly administered melatonin according to our chronobiotic protocol but only for shortterm, experienced a reappearance of their RBD symptoms over time. The observation that this group of patients converted at a similar rate as patients with the other known symptomatic mixture of treatment seems to indicate a necessity of at least 6 months melatonin treatment.

Only recently, various reports suggested sleep and the circadian timing system to be causally involved in neurodegenerative mechanisms including synucleinopathy (Leng et al., 2020; Hablitz et al., 2020). Over the last decade the previously unknown glymphatic system was described, elegantly explaining clearance of metabolic waste including synuclein from the brain during sleep (Xie et al., 2013; Holth et al., 2019; Fultz et al., 2019). Only this year, data in mice show that glymphatic influx and clearance exhibit endogenous, circadian rhythms (Hablitz et al., 2020). Circadian abnormalities and flattening of circadian rhythms have repeatedly been proven in patients with Parkinson`s disease (Videnovic et al., 2014; Leng et $a l ., 2020)$. Thus, the assumption that a synchronizing strengthening of circadian rhythms via light and melatonin - the hormone of darkness - may ameliorate neurodegenerative processes per se is tempting (Kunz, 2017; Videnovic et al., 2017).

Another interesting link exists between melatonin and Parkinson's disease. Patients treated with the beta2-adrenoreceptor antagonist propranolol are at an increased risk to develop Parkinson's disease, whereas patients treated with the beta2-adrenoreceptor agonist salbutamol are at a reduced risk (Mittal et al., 2017). As has been known for decades, lipophilic propranolol blocks melatonin secretion from the pineal gland via beta receptors (Cowen et al., 1985). Suppression of melatonin with beta blockers predominantly affects REM sleep, which can be reversed by exogenous melatonin (van den Heuvel et al., 1997). These mechanisms might explain the confounding effect of beta blockers in the first weeks of melatonin treatment in the observations presented here. The reported increased risk to develop Parkinson's disease is small in numbers. This may indicate that betablockers do not increase risk to develop Parkinson's disease per se, rather an ongoing process of neurodegeneration might be accelerated by reduced melatonin levels mediated by betablockers.

Two facts challenge our results: No previous research on the use of melatonin to treat RBD has reported similar effectiveness as observed in our cohort, and no evidence exists that the approximately 60 million Americans - predominantly the elderly - who have been using melatonin on a nightly basis at the crest of the "melatonin madness" in the mid-nineties (Reppert and Weaver, 1995) have experienced reduced rates of parkinsonism. Melatonin has 
been sold worldwide for the past 25 years as a hypnotic to be administered in connection with clock time independent events (e.g. "after a meal", "at bedtime"). Many, if not most people who administered melatonin will therefore not have adhered to a schedule based strictly on clock time. Another factor of importance is dosage. Because melatonin influences its own receptor (Gerdin et al., 2004), it is important to have a melatonin-free period over the day. Supraphysiologic melatonin doses, especially in low metabolizers, prevent the absence of melatonin during the day and could induce insensitivity in melatonin receptors the next evening (Braam et al., 2010).

Limitations: This is an observational study and the validity of observer-dependent results might therefore be questioned. Although all RBD symptom severity ratings were performed unblinded, they were based on bed-partner observation only and the changes in symptom severity were substantial. Symptoms such as frightening aggressiveness several times per week improved in almost all patients long-term treated with melatonin, replaced by milder symptoms like speaking aloud and slight movements occurring every other week. With regard to the primary outcome, phenoconversion also has been evaluated by referring neurologists, who were unaware of a possible disease-modifying effect of melatonin. Furthermore, low performers and patients suffering from NCD at baseline profited most according to objective neurocognitive tests. This makes systematic error in motor and cognitive performance scoring unlikely.

In conclusion, the present results strongly suggest that well-timed, long-term administration of melatonin to patients with $\mathrm{RRBD}$ is associated with reduced phenoconversion to parkinsonism and dementia and with improvement of specific clinical symptoms of prodromal synucleinopathies. Thus, melatonin may be associated with disease-modification in the prodromal state of synucleinopathies. 
medRxiv preprint doi: https://doi.org/10.1101/2020.11.05.20224592; this version posted November 12, 2020. The copyright holder for this preprint (which was not certified by peer review) is the author/funder, who has granted medRxiv a license to display the preprint in perpetuity.

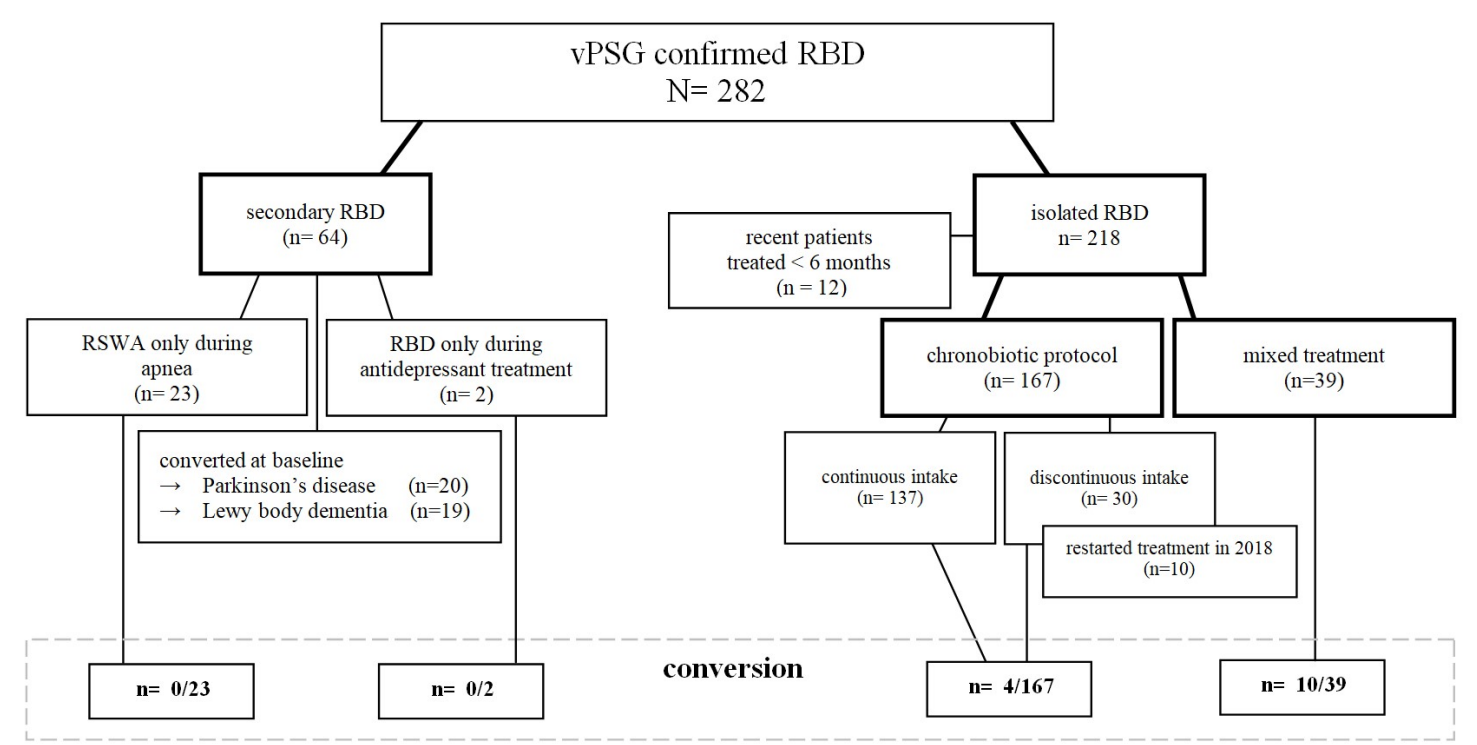

Figure 1. Patients with RBD Diagnosed 2008 - 2020

All RBD diagnoses confirmed by three night's vPSG; "recent patients" with iRBD were diagnosed in 2020 and therefore without 6 months of melatonin treatment or follow-up including CERAD and UPDRS. Patients with "discontinued intake" stopped melatonin intake mostly after 6 months. 
medRxiv preprint doi: https://doi.org/10.1101/2020.11.05.20224592; this version posted November 12, 2020. The copyright holder for this preprint (which was not certified by peer review) is the author/funder, who has granted medRxiv a license to display the preprint in perpetuity.

All rights reserved. No reuse allowed without permission.

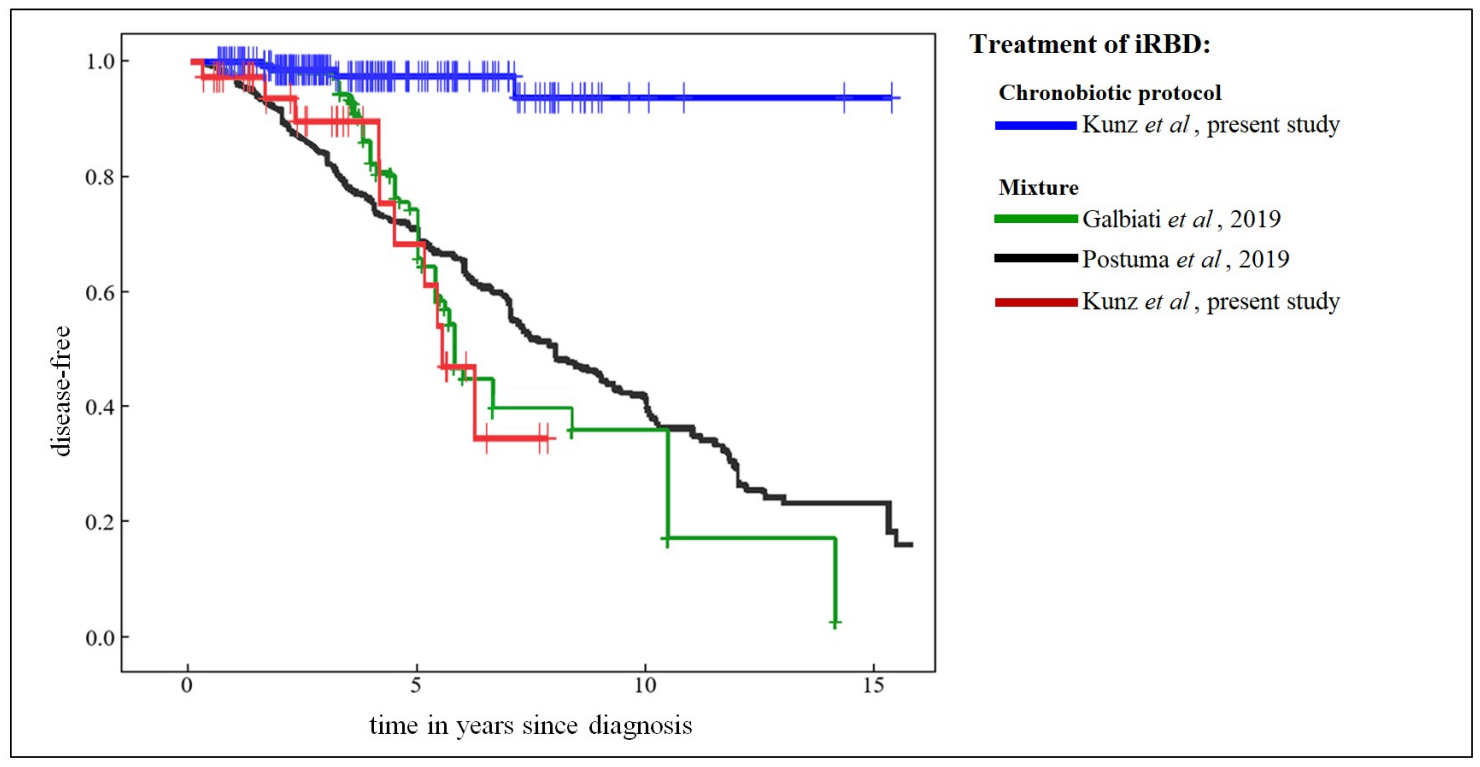

Figure 2. Phenoconversion from iRBD to Cinical Synucleinopathy

Kaplan-Meier curves, showing the cumulative disease-free survival. Right-censored events (vertical dashes) represent individual times of last follow-up, death or determination of conversion. To facilitate comparison, we depicted similar curves from two other published cohorts (Postuma et al., 2019; Galbiati et al., 2019 - with permission from Elsevier). 


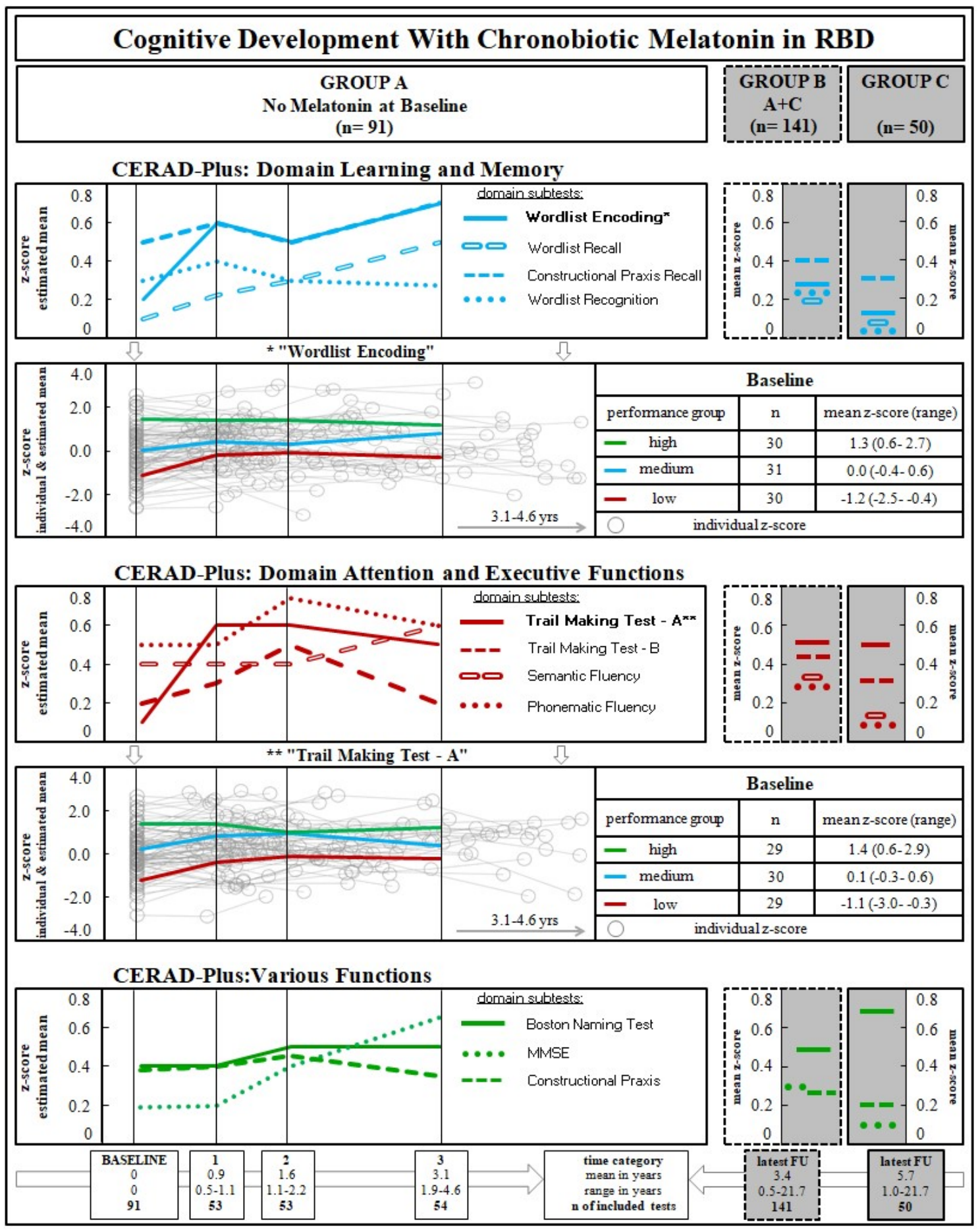

\section{Figure 3. Cognitive Development with Chronobiotic Melatonin in RBD}

CERAD-Plus subtests (total $\mathrm{n}=396$ ): results are $\mathrm{z}$-scores of all 11 subtests; arranged in 2 cognitive domains and "various functions"; z-scores are based on comparison with standard population, taking into account gender, age and years of education; a z-score " 0.0 " represents 
medRxiv preprint doi: https://doi.org/10.1101/2020.11.05.20224592; this version posted November 12, 2020. The copyright holder for this preprint (which was not certified by peer review) is the author/funder, who has granted medRxiv a license to display the preprint in perpetuity. All rights reserved. No reuse allowed without permission.

average norm value. Group A: Baseline and all follow-up data of patients without melatonin intake at baseline examination are shown; follow-up times are categorized including roughly equal numbers of tests taken (at $0.9,1.6$, and 3.1 years after baseline). Individual examination results (grey circles, connected by linear interpolation lines) are presented for tests with statistically significant (“*” and "***”) time and group effects ("Wordlist encoding" and "Trail Making Test-A");additional lines represent time courses of the categorized "high" (green), "medium" (blue), and "low" (red) performers at baseline. Group B: data at last follow-up of patients in groups $\mathrm{A}$ and $\mathrm{C}$ combined. Group C: data at last follow-up of patients who received melatonin already at baseline. 


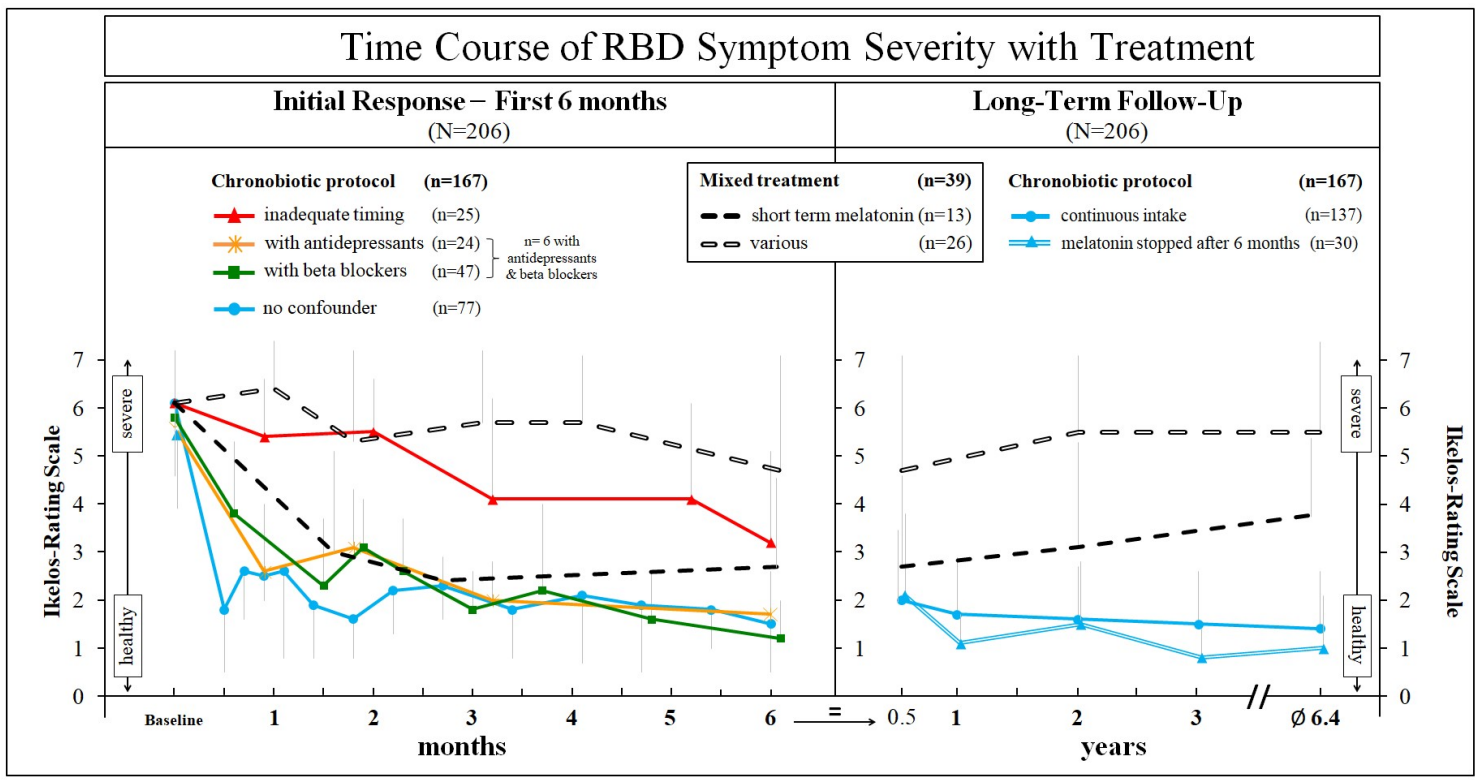

\section{Figure 4. Time Course of RBD Symptom Severity with Treatment}

Chronobiotic Protocol: $2 \mathrm{mg}$ melatonin slow-release, $\geq 6$ months, always-at-the-same-clocktime, between 10 and $11 \mathrm{pm}$ - corrected for chronotype, melatonin intake to be skipped in case impossible during this time-span; inadequate timing: initial administration with varying clock-times ("before bedtime") or too early ("after meal"); Mixed Treatment - short term melatonin: melatonin according to chronobiotic protocol but stopped after 1 to 3 months treatment; various: usual treatment of RBD at the advice of referring physician; Left panel: development of RBD symptom severity scores (Ikelos-RS) over the first 6 months; Right panel: symptom severity shown over subsequent years; for the chronobiotic protocol the former sub-groups are combined and now subdivided into a) one with continuous melatonin intake until last follow-up and b) one in which patients stopped melatonin intake after 6 months (patients felt "cured").

Ikelos-RS (total $n=1.498$ ): observational periods were either the last 6 months (default), or since the last interview if less than 6 months; sum (range 0 to 7) of scores "frequency" of RBD symptoms (never $=0, \leq 2$ to 3 times a month $=1,1$ to 2 times a week $=2,3$ to 5 times a week $=3$, daily $=4$ ) and "severity" (single most severe acting out: speech or slight distal movements $=0$, screaming or complex, non-aggressive movements $=1$, complex movements with risk of injury $=2$, leaving the bed $=3$ ). Ikelos ratings based on bed partner interview only; 8 patients had no regular bed partner at all times; for these patients, reports of friends/relatives during periods of e.g. vacation or visits were included. 
medRxiv preprint doi: https://doi.org/10.1101/2020.11.05.20224592; this version posted November 12, 2020. The copyright holder for this preprint (which was not certified by peer review) is the author/funder, who has granted medRxiv a license to display the preprint in perpetuity.

All rights reserved. No reuse allowed without permission.

Table 1. Generalizability of RBD Specifics

\begin{tabular}{|c|c|c|c|c|c|}
\hline & $\begin{array}{l}\text { Postuma et al., } \\
2019\end{array}$ & $\begin{array}{c}\text { Galbiati et al., } \\
2019\end{array}$ & $\begin{array}{c}\text { Fernández-Arcos et } \\
\text { al., 2016 }\end{array}$ & \multicolumn{2}{|c|}{$\begin{array}{l}\text { Kunz et al., } \\
\text { present study }\end{array}$} \\
\hline Study design & multicenter & $\begin{array}{l}\text { systematic review } \\
\text { and meta-analysis }\end{array}$ & single center & \multicolumn{2}{|c|}{ single center } \\
\hline Sample size N & 1280 & 3865 & 203 & 39 & 167 \\
\hline Treatment & mixed & mixed & mixed & mixed & chronobiotic protocol \\
\hline Male (\%) & 83 & $78(\mathrm{n}=2444)$ & 80 & 72 & 78 \\
\hline Age at estimated RBD onset (years) & - & - & $\mathrm{md}=63(40-81)$ & $61 \pm 12(23-84)$ & $64 \pm 9(39-84)$ \\
\hline Age at baseline/RBD diagnosis (years) & $66 \pm 8$ & $68 \pm 3(\mathrm{n}=2444)$ & $\mathrm{md}=68(50-85)$ & $66 \pm 11(30-86)$ & $69 \pm 8(41-86)$ \\
\hline RBD severity: falling out of bed (\%) & - & - & 77 & 64 & 62 \\
\hline Obstructive sleep apnea (\%) & 27 & - & 12 & 34 & 27 \\
\hline Excessive daytime sleepiness (ESS) & - & - & $8.3 \pm 4.7$ & $7.4 \pm 4.5$ & $7.1 \pm 4.0$ \\
\hline $\begin{array}{l}\text { Beta blockers (\%) } \\
\text { Antidepressants (\%) }\end{array}$ & $\begin{array}{l}- \\
-\end{array}$ & $\begin{array}{l}- \\
-\end{array}$ & $\overline{26}$ & $\begin{array}{l}26 \\
31\end{array}$ & $\begin{array}{l}33 \\
18\end{array}$ \\
\hline DaT-SPECT abnormal (\%) & $44(\mathrm{n}=245)$ & - & - & $30(\mathrm{n}=10)$ & $35\left(\mathrm{n}=117^{* \mathrm{c}}\right)$ \\
\hline UPDRS-III & $\begin{array}{r}6.26 \pm 4.94(\mathrm{n}=57)^{* \mathrm{a}} \\
3.12 \pm 3.82(\mathrm{n}=299)^{* \mathrm{~b}}\end{array}$ & - & - & $1.0 \pm 2.0(\mathrm{n}=17)$ & $2.1 \pm 3.1(\mathrm{n}=81)$ \\
\hline MMSE & $\begin{array}{l}26.9 \pm 3.4(\mathrm{n}=132)^{*{ }^{* a}} \\
28.1 \pm 1.8(\mathrm{n}=375)^{* \mathrm{~b}}\end{array}$ & - & - & $29.0 \pm 0.9(\mathrm{n}=16)$ & $29.2 \pm 1.0(\mathrm{n}=99)$ \\
\hline $\begin{array}{ll}\text { Olfaction abnormal (\%) } & \begin{array}{l}\text { hyposmia } \\
\text { anosmia }\end{array} \\
\end{array}$ & $67(\mathrm{n}=628)$ & - & - & $\begin{array}{ll}47 & (\mathrm{n}=15) \\
47 & \end{array}$ & $\begin{array}{ll}41 & (\mathrm{n}=96) \\
56 & \end{array}$ \\
\hline Constipation/gastrointestinal dysfunction (\%) & $43(n=830)$ & - & - & $43(n=14)$ & $47(\mathrm{n}=38)$ \\
\hline Urinary dysfunction (\%) & $31(\mathrm{n}=687)$ & - & - & $57(\mathrm{n}=14)$ & $51(\mathrm{n}=37)$ \\
\hline Sexual dysfunction (\%) & $43(n=276)$ & - & - & $63(\mathrm{n}=8)$ & $59(\mathrm{n}=29)$ \\
\hline Average follow up (years) & $3.6(\max .19)$ & $4.75 \pm 2.43$ & $\mathrm{md}=5(0.1-17)$ & $3.1 \pm 2.1(0.3-7.6)$ & $4.2 \pm 3.1(0.6-21.7)$ \\
\hline Conversion \% (n) & $28(352)$ & $31.9(1235)$ & $34(69)$ & $25.6(10)$ & $2.4(4)$ \\
\hline Conversion-rate per year (\%) & 6.3 & 6.7 & 6.8 & 8.3 & 0.6 \\
\hline
\end{tabular}

Demographics and RBD-specific clinical data of our cohort and of three representative cohorts including neuromotor (UPDRS-III) and cognitive performance (Mini Mental Status Examination - MMSE), olfactory ability (Sniffin' Sticks), autonomic functioning (SCOPAAUT), and dopamine-transporter (DaT)-SPECT. Numbers are given in percent, mean values with standard deviations, and medians (md) with ranges. Numbers are before melatonin treatment, except for DaTSCAN for which 40 patients were scanned after initiation of melatonin treatment. *a: developed disease. ${ }^{*}$ b: disease free. ${ }^{*} \mathrm{c}: 40 / 117$ had DaT-SPECT during treatment. 
medRxiv preprint doi: https://doi.org/10.1101/2020.11.05.20224592; this version posted November 12, 2020. The copyright holder for this

\section{References}

Aurora RN, Zak RS, Maganti RK, Auerbach SH, Casey KR, Chowdhuri S, et al. Standards of Practice Committee; American Academy of Sleep Medicine: Best practice guide for the treatment of REM sleep behavior disorder (RBD). J Clin Sleep Med 2010; 6: 85-95.

Bes FW, Jobert M, Müller C, Schulz H. The diurnal distribution of sleep propensity: experimental data about the interaction of the propensities for slow-wave sleep and REM sleep. J Sleep Res 1996; 5: 90-8.

Braak H, Del Tredici K, Rub U, de Vos RA, Jansen Steur EN, Braak E. Staging of brain pathology related to sporadic Parkinson's disease. Neurobiol Aging 2003; 24: 197-211.

Braam W, Van Geijlswijk I, Keijzer H, Smits M, Didden R, Curfs L. Loss of response to melatonin treatment is associated with slow melatonin metabolism. J Intell Disability Res 2010; 54: 547-55.

Cardinali DP. Melatonin: Clinical perspectives in neurodegeneration. Front Endocrinol 2019; 10: 480. doi:10.3389/fendo.2019.00480

Cowen PJ, Bevan JS, Gosden B, Elliott SA. Treatment with beta-adrenoceptor blockers reduces plasma melatonin concentration. Br J Clin Pharmacol 1985; 19: 258-60.

Darweesh SKL, Wolters FJ, Postuma RB, Stricker BH, Hofman A, Koudstaal PJ, et al. Association Between Poor Cognitive Functioning and Risk of Incident Parkinsonism: The Rotterdam Study. JAMA Neurol 2017; 74: 1431-8.

Dawson D, Armstrong SM. Chronobiotics - drugs that shift rhythms. Pharmacol Ther 1996; 69: $15-36$.

Dijk DJ, Czeisler CA. Contribution of the circadian pacemaker and the sleep homeostat to sleep propensity, sleep structure, electroencephalographic slow waves, and sleep spindle activity in humans. J Neurosci 1995; 15: 3526-38.

Djang DSW, Janssen MJR, Bohnen N, et al. SNM practice guideline for dopamine transporter imaging with ${ }^{123}$ I-Ioflupane SPECT 1.0. J Nucl Med 2012; 53:154-163.

Espay AJ, Brundin P, Lang AE. Precision medicine for disease modification in Parkinson disease. Nat Rev Neurol 2017; 13: 119-26.

Espay AJ, Kalia LV, Gan-Or Z, Williams-Gray CH, Bedard PL, Rowe SM, et al. Disease modification and biomarker development in Parkinson disease: Revision or reconstruction? Neurol 2020; 94: 1-14. doi:10.1212/WNL.0000000000009107

Fernandez-Arcos A, Iranzo A, Serradell M, Gaig C, Santamaria J. The Clinical Phenotype of Idiopathic Rapid Eye Movement Sleep Behavior Disorder at Presentation: A Study in 203 Consecutive Patients. Sleep 2016; 39: 121-32. 
Fultz NE, Bonmassar G, Setsompop K, et al. Coupled electrophysiological, hemodynamic, and cerebrospinal fluid oscillations in human sleep. Science 2019; 366: 628-631.

Galbiati A, Verga L, Giora E, Zucconi M, Ferini-Strambi L. The risk of neurodegeneration in REM sleep behavior disorder: A systematic review and meta-analysis of longitudinal studies. Sleep Med Rev 2019; 43: 37-46.

GBD 2016 Collaborators Neurology. Global, regional, and national burden of neurological disorders, 1990-2016: a systematic analysis for the Global Burden of Disease Study 2016. Lancet Neurol 2019; 18: 459-80.

Genier-Marchand D, Montplaisir J, Postuma RB, Rahayel S, Gagnon JF. Detecting the Cognitive Prodrome of Dementia with Lewy Bodies: A Prospective Study of REM Sleep Behavior Disorder. Sleep 2017; 40: 1-11.

Gerdin MJ, Masana MI, Rivera-Bermudez MA, Hudson RL, Earnest DJ, Gilette MU, Dubocovich ML. Melatonin desensitizes endogenous MT2 melatonin receptors in the rat suprachiasmatic nucleus: relevance for defining the periods of sensitivity of the mammalian circadian clock to melatonin. FASEB J 2004; 18: 1646-56.

Gillette MU, McArthur AJ. Circadian actions of melatonin at the suprachiasmatic nucleus. Behav Brain Res 1996; 73: 135-9.

Goetz CG, Tilley BC, Shaftman SR, et al. Movement Disorder Society-sponsored revision of the Unified Parkinson's Disease Rating-scale (MDS-UPDRS): scale presentation and clinimetric testing results. Mov Disord 2008; 23: 2129-70.

Hablitz LM, Plá V, Gianetto M, Vinitsky HS, Stæger FF, Metcalfe T, et al. Circadian control of brain glymphatic and lymphatic fluid flow. Nature Comm 2020; 11: 4411. doi.org/10.1038/s41467-020-18115-2.

Holth JK, Fritschi SK, Wang C, Pedersen NP, Cirrito JR, Mahan TE, et al. The sleep-wake cycle regulates brain interstitial fluid tau in mice and CSF tau in humans. Science 2019; 363: 880-4.

Hummel T, Sekinger B, Wolf SR, Pauli E, Kobal G. 'Sniffin' Sticks': olfactory performance assessed by the combined testing of odour identification, odor discrimination and olfactory threshold. Chem Senses 1997; 22: 39-52.

Iranzo A, Fernandez-Arcos A, Tolosa E, Serradell M, Molinuevo JL, Valldeoriola F, et al. Neurodegenerative disorder risk in idiopathic REM sleep behavior disorder: study in 174 patients. PLoS One 2014; 9: e89741. 
medRxiv preprint doi: https://doi.org/10.1101/2020.11.05.20224592; this version posted November 12, 2020. The copyright holder for this

Iranzo A, Santamaria J, Tolosa E. Idiopathic rapid eye movement sleep behaviour disorder: diagnosis, management, and the need for neuroprotective interventions. Lancet Neurol 2016; 15: 405-19.

Iranzo A, Santamaria J, Valldeoriola F, Serradell M, Salamero M, Gaig C, et al. Dopamine transporter imaging deficit predicts early transition to synucleinopathy in idiopathic rapid eye movement sleep behavior disorder. Ann Neurol 2017; 82: 419-28.

Jockers R, Delagrange P, Dubocovich ML, Markus RP, Renault N, Tosini G, et al. Update on melatonin receptors: IUPHAR Review 20. British J Pharmacol 2016; 173: 2702-25.

Kunz D, Bes F. Melatonin effects in a patient with severe REM sleep behavior disorder: case report and theoretical considerations. Neuropsychobiology 1997; 36: 211-4.

Kunz D, Bes F. Melatonin as a therapy in REM sleep behavior disorder patients: an openlabeled pilot study on the possible influence of melatonin on REM-sleep regulation. Mov Disord 1999; 14: 507-11.

Kunz D, Bes F. Exogenous melatonin in periodic limb movement disorder: an open clinical trial and a hypothesis. Sleep 2001; 24: 183-187.

Kunz D, Mahlberg R, Müller C, Tilmann A, Bes F. Melatonin in patients with reduced REM sleep duration: two randomized controlled trials. J Clin Endocrinol Metab 2004; 89: 128 34.

Kunz D. Chronobiotic Protocol and Circadian Sleep Propensity Index: New Tools for Clinical Routine and Research on Melatonin and Sleep. Pharmacopsychiatry 2004; 37:139-146.

Kunz D, Mahlberg R. A two-part, double-blind, placebo-controlled trial of exogenous melatonin in REM sleep behaviour disorder. J Sleep Res. 2010; 19: 591-6.

Kunz D. Circadian Rhythms are Everywhere: Except in Neurodegenerative Disorders. Curr Alzheimer Res 2017; 14: 1018-1021.

Leng Y, Blackwell T, Cawthon PM, Ancoli-Israel S, Stone KL, Yaffe K. Association of Circadian Abnormalities in Older Adults With An Increased Risk of Developing Parkinson Disease. JAMA Neurol 2020; doi:10.1001/jamaneurol.2020.1623.

Lewy AJ, Ahmed S, Jackson JM, Sack RL. Melatonin shifts human circadian rhythms according to a phase-response curve. Chronobiol Int 1992; 9: 380-92.

Mittal S, Bjornevik K, Im DS, Flierl A, Dong X, Locascio JJ, et al. beta2-Adrenoreceptor is a regulator of the alpha-synuclein gene driving risk of Parkinson's disease. Science 2017; 357: 891-8.

Monk TH, Buysse DJ, Carrier J, Kupfer DJ. Inducing jet-lag in older people: Directional assymetry. J Sleep Res 2000; 9: 101-16. 
medRxiv preprint doi: https://doi.org/10.1101/2020.11.05.20224592; this version posted November 12, 2020. The copyright holder for this

Postuma RB, Berg D. Prodromal Parkinson's Disease: The Decade Past, the Decade to Come. Mov Disord 2019; 34: 665-75.

Postuma RB, Iranzo A, Hu M, Högl B, Boeve BF, Manni R et al. Risk and predictors of dementia and parkinsonism in idiopathic REM sleep behaviour disorder: a multicentre study. Brain 2019; 142: 744-59.

Reiter RJ, Tan D, Rosales-Coral S, Manchester LC: The universal nature, unequal distribution and antioxidant functions of melatonin and its derivatives. Mini-Rev Med Chem 2013; 13 : $373-84$.

Reppert S and Weaver D. Melatonin madness. Cell 1995; 83: 1059-62.

Samel A, Wegmann HM, Vejvoda M, Maass H, Gundel A, Schutz M. Influence of melatonin treatment on human circadian rhythmicity before and after a simulated 9-hr time shift. J Biol Rhythms 1991; 6: 235-48.

Saper C B, Scammell T E, Lu J. Hypothalamic regulation of sleep and circadian rhythms. Nature 2005; 437: 1257-1263.

Schenck CH, Bundlie SR, Ettinger MG, Mahowald MW. Chronic behavioral disorders of human REM sleep: a new category of parasomnia. Sleep 1986; 9: 293-308.

Schenck CH, Bundlie SR, Mahowald MW. Delayed emergence of a parkinsonian disorder in $38 \%$ of 29 older men initially diagnosed with idiopathic rapid eye movement sleep behaviour disorder. Neurology 1996; 46: 388-93.

Schenck CH, Boeve BF, Mahowald MW. Delayed emergence of a parkinsonian disorder or dementia in $81 \%$ of older men initially diagnosed with idiopathic rapid eye movement sleep behavior disorder: a 16-year update on a previously reported series. Sleep Med 2013; 14: $744-8$.

Schmid NS, Ehrensperger MM, Berres M, Beck IR, Monsch AU. The extension of the German CERAD-Neuropsychological Assessment Battery with tests assessing subcortical, executive and frontal functions improves accuracy in dementia diagnosis. Geriatr Cogn Disord Extra 2014; 4: 322-34.

Terzaghi M, Zucchella C, Rustioni V, Sinforiani E, Manni R. Cognitive performances and mild cognitive impairment in idiopathic rapid eye movement sleep behavior disorder: results of a longitudinal follow-up study. Sleep 2013; 36: 1527-32.

Utiger RD. Melatonin - the hormone of darkness. N Engl J Med 1992; 327: 1377-9.

Van Den Heuvel CJ, Reid KJ, Dawson D. Effect of atenolol on nocturnal sleep and temperature in young men: reversal by pharmacological doses of melatonin. Physiol Behav 1997; 61: 795-802. 
medRxiv preprint doi: https://doi.org/10.1101/2020.11.05.20224592; this version posted November 12, 2020. The copyright holder for this preprint (which was not certified by peer review) is the author/funder, who has granted medRxiv a license to display the preprint in perpetuity. All rights reserved. No reuse allowed without permission.

Videnovic A, Noble C, Reid KJ, Peng J, Turek FW, Marconi A, et al. Circadian melatonin rhythm and excessive daytime sleepiness in Parkinson`s disease. JAMA Neurol 2014; 71: 463-9.

Videnovic A, Klerman EB, Wang W, Marconi A, Kuhta T, Zee PC. Timed light therapy for sleep and daytime sleepiness associated with parkinson disease: a randomized clinical trial. JAMA Neurol 2017; 74: 411-8.

Videnovic A, Ju Y-ES, Arnulf I, Cochen-DeCock V, Högl B, Kunz D, et al. Clinical trials in REM Sleep Behavior Disorder - Challenges and Opportunities. J Neurol Neurosurg Psychiatry 2020; doi:10.1136/jnnp-2020-322875.

Visser M, Marinus J, Stiggelbout AM, van Hilten JJ. Assessment of autonomic dysfunction in Parkinson's disease: the SCOPA-AUT. Mov Disord 2004; 19: 1306-12.

Xie L, Kang H, Xu Q, Chen MJ,Liao M, et al. Sleep drives metabolite clearance from the brain. Science 2013; 342: 373-7. 